\title{
Preparing a High-Quality and Impactful Sport Science Manuscript
}

Research impact can be defined in the context of sports performance and physiology as the contribution to both the research/academic (knowledge discovery) and sporting (real-world application) communities. Practical application has always been a hallmark of the International Journal of Sports Physiology and Performance $(I J S P P)$, with articles that clearly identify how and why study outcomes can be implemented in training and competition to enhance performance. Some outcomes apply to a broad range of sports, while others are more specific to a sporting discipline, such as aquatic sports, court or field sports, individual time-based sports, or otherwise just meet the requirements of a single sport, discipline, or event. Strengthening the practice and reporting of sport and exercise science research is the responsibility of every author. ${ }^{1}$ Three key areas in ensuring a high-quality manuscript are project formulation and design; methodology and analytical issues; and style, clarity, and quality of the written presentation.

It can be instructive to examine other scientific, allied health, and medical journals for insights on experimental design and analysis, with reference to so-called implementation research. It is often challenging to implement the results of experimental research, and authors need to clearly identify how their work can be applied in sport. Results from a randomized controlled trial are not always easily implemented, as differences between laboratory and field conditions, between individual and team sports, and between various competition formats can make translation difficult. Within-cluster repeated-measure designs can be used to control variance between intervention and control groups by allowing the same clusters to receive a designated sequence of interventions or conditions. Novel application of counterbalanced designs has been proposed in clinical research where repeated measures are employed through the elegant use of a crossover to test the implementation strategy. ${ }^{2}$

The notion of individual responders to exercise and training interventions is self-evident to athletes, coaches, and researchers. The challenge is how to address this issue during the statistical analyses and account for measurement error, sampling variation, true interindividual differences, and the frequency of nonresponders. ${ }^{3}$ Various analytical approaches for detailing individual responses are emerging and warrant close consideration. Quantifying individual responses in a controlled trial requires a large sample size or averaging of repeated measurements to compensate for a large error of measurement. ${ }^{4}$ The mean and SD of change scores should be reported for all treatment groups. However, authors should avoid the common temptation to revert to individual responders simply because the main effects are unclear.

Sport scientists are interested in the mechanisms of effects, as well as their identification and description. The extent to which a mechanistic variable mediates an effect through measurements that link the predictor to the dependent variable adds to the impact of a study. ${ }^{3}$ Many sport and exercise studies lend themselves for adjustment to predictor variables, such as training history, fitness level $\left(\mathrm{VO}_{2} \mathrm{max}\right)$, or an anthropometric characteristic (body mass). For exercise and training interventions, a plot of change scores of the dependent variable versus those of potential mediators can be useful. Mechanism variables are particularly useful in unblinded interventions common in sport science research to differentiate the underlying effect of the intervention from that of a placebo or nocebo effect. ${ }^{5}$

Two common questions in scientific research are "How does the intervention work?" and "For which groups does the intervention work?" These questions are clearly important in sport science research for researchers, sport science practitioners, and coaches (particularly, the second question). Mediating variables can be defined as those that describe the process by which the intervention achieves its effects, while moderating variables yield a different effect at different values of the moderating variable. Ideally, mediating and moderating variables should be considered a priori during the study formulation, although they often emerge during the analysis of the experimental data. Authors should also be mindful of confounding variables, where an outside influence changes the effect of a dependent and independent variable. An obvious example is the possible effect(s) of environmental conditions (ambient temperature, humidity, or wind) that could influence field-based sport performance.

Apart from the more scientific elements of a published article, the issues of style, clarity, and conciseness of the written presentation are critical and often differentiate the high-quality articles from average articles. The style requirements in the Authorship Guidelines for IJSPP indicate that manuscripts should be written in first person using the active voice. First-person prose involves use of " $\mathrm{I}$ " in the single form and "We" in the plural. In academic writing, an occasional "I" or "We" can be appropriate, although best not overdone.

Writing should be concise and direct. Avoid using unnecessary jargon and abbreviations, but inclusion of an acronym or abbreviation is sensible if it is more commonly recognized than the fully writtenout version of a term. Measurements of length, height, mass, and volume should be reported in metric units (meters and kilograms). Excessive use of abbreviations is a common irritation for readers, and authors are encouraged to use them in a parsimonious manner. Only standard physiological abbreviations should be used because nonstandard abbreviations are unnecessary and confusing. Typographical errors, spelling errors, and grammar errors are obvious but often overlooked. Turgid prose is the bane of all readers and reviewersbest avoided by careful and detailed preparation, editing, and checking of drafts by all coauthors prior to submission. Authors should always be creative with their narrative, and reviewers, constructive rather than instructive and dogmatic.

David Pyne, IJSPP Associate Editor, University of Canberra, Australia

\section{References}

1. Halperin I, Vigotsky AD, Foster C, Pyne DB. Strengthening the practice of exercise and sport-science research. Int J Sports Physiol Perform. 2018;13(2):127-134. PubMed ID: 28787228 doi:10.1123/ ijspp.2017-0322 
2. Hecksteden A, Pitsch W, Rosenberger F, Meyer T. Repeated testing for the assessment of individual response to exercise training. $J$ Appl Physiol. 2018;124(6):1567-1579. PubMed ID: 29357481 doi:10. 1152/japplphysiol.00896.2017

3. Hopkins WG. Individual responses made easy. J Appl Physiol. 2015;118(12):1444-1446. PubMed ID: 25678695 doi:10.1152/ japplphysiol.00098.2015
4. Mackinnon DP. Integrating mediators and moderators in research design. Res Soc Work Pract. 2011;21(6):675-681. PubMed ID: 22675239 doi:10.1177/1049731511414148

5. Hopkins WG, Marshall SW, Batterham AM, Hanin J. Progressive statistics for studies in sports medicine and exercise science. Med Sci Sports Exerc. 2009;41(1):3-13. PubMed ID: 19092709 doi:10.1249/ MSS.0b013e31818cb278 